\title{
The Development of a Program System for a Design of Superconducting Poloidal Field Coils
}

\author{
S. Tado, T. Narikawa, H. Tomita, S. Yamaguchi, T. Tsukamoto, K. Ueda, T. Yamada, \\ T. Fukunaga, T. Sato, T. Ichihara, O. Asai, Y. Hattori, S. Sakabe \\ Mitsubishi Electric Corporation, 2-2-3, Marunouchi, Chiyoda-ku, Tokyo, Japan
}

Abstract

A program system was developed for the purpose of the computer aided design of the superconducting poloidal coils in a tokamak. The program system consists of a main program, subroutines and data files. The main program effects the initialization of the program system and subsequent control of calculation process. The system is operated both in interactive mode and batch one by modifying it. The subroutines perform the calculation for electromagnetic variables, the structural design of the coils and the estimation for their design solutions. The data files play the role of a data base.

The program system greatly reduces the large amount of labor and time required to design the superconducting poloidal colls. Utilizing the data files, users can readily redesign any part of the coils.

\section{Introduction}

There is strict space requirement for each part, such as a coil, plasma vacuum chamber and blanket in tokamak. Then, superconducting poloidal coils must also be designed under spatial restraint.

It is necessary to find out the optimum design solutions of the coils concerning their construction to endure heavy loads caused by electromagnetic force and their superconductor under high magnetic field. However, it is troublesome to find oit such solutions under the spatially limited condition. Accordingly, the optimum design solutions of superconducting poloidal coils of tokamak are generally searched by the repetition of the design process based on the design flow shown in Fig. 1.

In the design, there is need to pick out values such as maxima in large amounts of calculations obtained from the analyses of the magnetic field and the electromagnetic force. Moreover, the repeating calculation may be required so that design variables cannot be uniquely determined owing to their correlation. These calculation procedures are very trouble and require the large amount of labor and time. Therefore, a computer aided design system is demanded in order to-carry out the design calculation efficiently.

Although there are many programs to calculate magnetic field, electromagnetic force and coil inductance, a program system to make the design of the superconducting poloidal coils could not be found. Therefore, the demand for such program system is on the increase with the development of research in tokamak. 
In the present paper, we describe the outline and the functions of the computer program system developed for designing superconducting poloidal coils in tokamak.

2. Features and Function of the Computer Program System

The program system is written in FORTRAN. Figure 2 shows the constitution of the system. It consists of a main program, subroutines and data flles.

The main program effects the initialization of the system and subsequent control of calculation process. The user is able to accomplish his design in sequence in interactive mode. Also this system can be operated in batch mode by rewriting the main program according to the calculation process in the

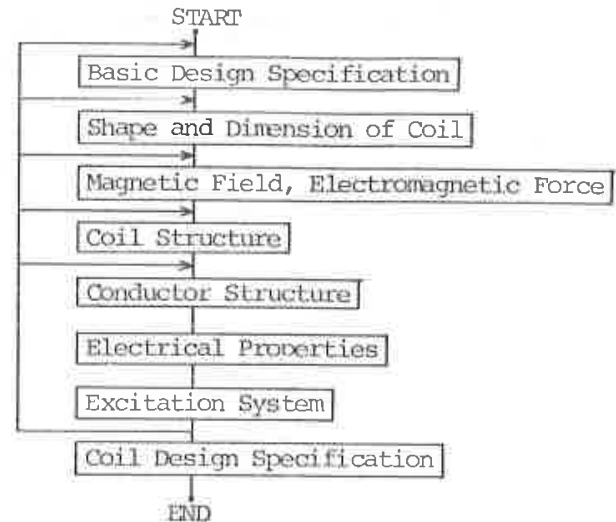

Fig. 1 Design flow of superconducting coil required design flow. Consequently, it is ready to make the design in which iterative calculation must be carried out.

The subroutines perform the calculation for electromagnetic variables, the structural design of the coils and the estimation for their design solutions. In every subroutine, the data used in the calculation of the design variables are directly read from the data files, and, after calculation and output, the results are written into the data files automatically, Every subroutine has a closed form. Consequently, it is ready to revise and/or extend its function. The default values of the design variables are set as much as possible, which the user may change. Then, even a user who does not completely know the design of superconducting poloidal coils may accomplish the design of the coils at a desirable technical level, which is very effective in case studies of poloidal coils relating to the total design study of tokamak.

All the calculation results are stored in the data files so that the redesign owing to partial design modification is readily accomplished. Moreover, the calculation results are output in figures and tables so that the user can quickly and easily examine the results provided by the system.

\section{Discription of the Subroutines}

'The finer details of the subroutines included in the program system are explained below.

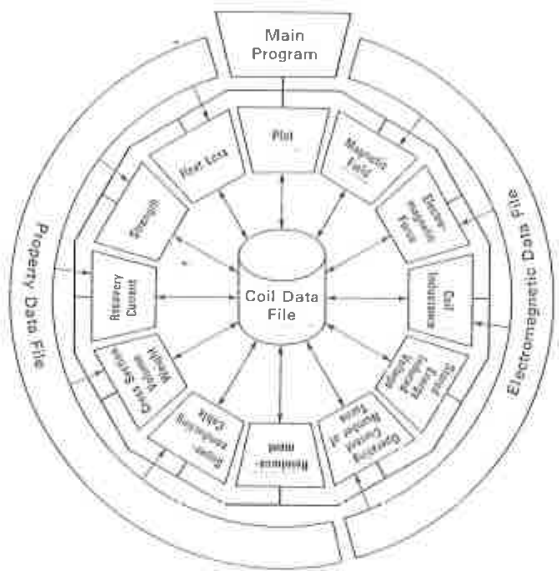

Fig. 2 Constitution of program system

\subsection{Plot}

This subroutine plots the position and shape of the poloidal coils from the input data stored in the data file. With the aid of this routine, the position and shape of the coils 
may be readily checked.

\subsection{Magnetic Fie1d}

This subroutine calculates the magnetic flux density, $\vec{B}$, and it's change rate with time, $\overline{\dot{B}}$, in the cross section of the coil. Additionally, this routine picks out the maximum values of $B$ and $\vec{B}$, and integrates $\vec{B}$ over the conductor domain.

When the $i-t h$ coil is graded into $k_{i}$ parts and the $l$-th part is excited by Ampere turns, $I_{i, l}\left(t_{m}\right)$, at the instant of time, $t_{m}$, the magnetic flux density, $\vec{B}_{j}\left(t_{m}\right)$, and its change rate with time, $\vec{B}_{j}\left(t_{m}\right)$, at the $j-t h$ calculating point, can be determined from the following formulae:

$$
\begin{aligned}
\vec{B}_{j}\left(t_{m}\right)= & \sum_{i=1}^{n} \sum_{l=1}^{k_{i}} \vec{b}_{i, l, j} I_{i, l}\left(t_{m}\right) \\
\vec{B}_{j}\left(t_{m}\right)= & \frac{1}{t_{m+1}-t_{m}} \sum_{i=1}^{n} \sum_{l=1}^{k} \vec{b}_{i, l, j}\left\{I_{i, l}\left(t_{m+l}\right)-I_{i, \ell}\left(t_{m}\right)\right\} \\
& \text { where } I_{i}\left(t_{m}\right)=\sum_{l=1}^{k_{i}} I_{i, l}\left(t_{m}\right)
\end{aligned}
$$

where $\vec{b}_{i, \ell, j}$ is the normalized magnetic flux density generated at the $j$-th calculating point by the $\boldsymbol{\ell}$-th graded part of the $i-t h$ coil. The values of $\vec{B}$ and $\vec{B}$ is readily calculated for various operating conditions of the coils using the calculating method mentioned above.

\subsection{Electromagnetic Force}

This subroutine calculates the electromagnetic force acting on the coils.

The cross section of the 1-th graded part of the $i-t h$ coil is devided into mxn rectangular elements. Then, the electromagnetic force acting on the conductor per unit length along the current path of the $i-t h \operatorname{coil}, \vec{F}_{i}(t)$, is given by the following equation:

$$
\bar{F}_{i}(t)=\sum_{\ell=1}^{k_{j}} \sum_{j=1}^{m \times n} \frac{1}{m \times n_{\ell}} I_{i, \ell}(t)\left[\vec{i}_{\ell, j} \times \vec{B}_{1, \ell, j}(t)\right]
$$

where $\vec{i}_{\ell, j}$ is current path vector.

When the ripple field generated by the toroidal coils is negligibly small, the electromagnetic force is unfform along the current path. But, in general, it is not uniform and the bending moment is caused by this ripple field. Then, in this routine, such force is calculated by applying Eq. (4) to each segment which is devided along the current path.

\subsection{Inductance}

This subroutine calculates the self Inductance, the mutual inductance and the coupling coefficient between the the pololdal coll, including the plasma. The inductance is calculated as the inductance of a one-turn coil and is output in the form of a inductance matrix. When two or more coils are connected in series in a poloidal coil system, the calculation is performed by regarding the coil group as a coil.

\subsection{Stored Energy and Induced Voltage}

This subroutine calculates the stored energy and the induced voltage of each coil. The stored energy of the i-th coil-group is given by the following equation:

$$
\ddot{E}_{i}(t)=\frac{1}{2} \sum_{k=1}^{k} \sum_{j=1}^{n} M_{k j} I_{k}(t) I_{j}(t)
$$

where $M_{k j}$ is the coil inductance between the $k-t h$ coll and the $j-t h$ one and $k$ is the number of the colls belong to the i-th coll-group. 
The voltage induced in one turn of the k-th coil is given by the following equation:

$$
v_{k}(t)=\sum_{j=1}^{n} M_{k j} \frac{I_{j}\left(t_{m+1}\right)-I_{j}\left(t_{m}\right)}{t_{m+1}-t_{m}}
$$

3.6 Operating Current and Number of Turns

This subroutine interactively determines the operation current and the number of turns of every coil. The design method to determine the optimum values of these variables has not been fully established. In this routine, the user decides on the operating current of each coil according to the procedure mentioned below.

In view of coil protection on quench, the operating current giving the muximum current density in the conductor, $I_{o p}$, is given by $I=a E^{b}$, where $\mathrm{I}$ is the stored energy, a and $b$ constants [1]. On the other hand, $I_{\text {op }}$ is given on the basis of past records of the operating current. Then, comparing these two values, the user interactively chooses and inputs one of them. The number of turns and induced voltage are calculated from it automatically. The user can revise the values of $I_{\text {op }}$ after computation.

\subsection{Reinforcement}

This subroutine firstly calculates the area of cross section of the reinforcement to keep the hoop stress of the conductor below the allowable value under the maximum electromagnetic force. If the result of the calculation is larger than the total area of cross section of the coil, the result means the solution of structural design is not exist. Therefore, redesign is required for some parts of the former design stage.

Second, the dimension of each part of the reinforcement is to be designed using the results of the required area of cross section of the structure. In this routine, typical four types of structures are prepared for design of the reinforcement. For each type, the the dimensions of the structure will automatically be decided from minimum data input.

Finally, the user could optionally modify some dimensions obtained by the processes mentioned above in manual mode.

\subsection{Superconducting Cable}

This subroutine determines the number of the strands and the dimensions of the superconducting filament, CuNi sheath and stabilizing Cu matrix which compose the conductor shown Fig. 3. The procedure in this routine is as follows:

The sectional area of the superconducting filaments of a conductor required for given operating current, $S_{1}$, is calculated. The number and the diameter of the strand are determined on the basis of the dimensions of the cross section occupied by the conductor. Next, another sectionalarea of the filaments of a conductor, $S_{2}$, is calculaced from the dimensions of the filament, sheath and Gu matrix input by the user. Comparing two values, the user modifies the dimensions of the filament, sheath and cu matrix until the condition of $S_{2} \gtreqless S_{1}$ is satisfied. Finally, the cooling perimeter and the sectional area of the stbilizing copper matrix are calculated.

3.9 Cross Section, Volume and Weight

This subroutine calculates the sectional area, volume and weight of the components material, such as can, insulator, conductor, reinforcement and helium. These values are 
necessary to calculate the heat loss and cooling capacity of the coil. The average current density is also provided in this routine.

\subsection{Recovery Current}

This subroutine calculates the recovery current, $I_{r}$, in the bath-cooling by giving the maximum magnetic field, B ${ }_{\text {max }}$, the operating current, $I_{\text {op }}$, the sectional area of the stabilizing copper matrix, $A$, the cooling perimeter, $p$, and the cooling efficiency, $n$, which are provided by the previous subroutines. The recovery current is given by following equation:

$$
I_{Y}=\sqrt{\frac{\rho A q_{e} \eta}{\rho}}
$$

where $\rho$ is the resistivity of the stabilizing copper, which varies with magnetic flux density. The heat flux on normalization, $\mathrm{q}_{\mathrm{e}}$, is obtained on Maddock's "equal area criterion" [2].

\subsection{Strength}

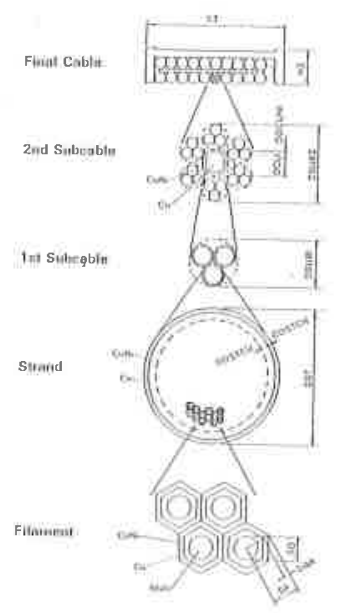

Fig. 3 Structure of conductor

This subroutine analyzes the structure of the coil to be designed against the electromagnetic force. Hoop stress, bending stress and allowable buckling load are calculated and, additionally, the safety factor in each loading condition is given in this routine.

The hoop stress, bending stress and allowable bucking load are obtained from the following approximate formulae[3], i.e.

$$
\begin{aligned}
& \text { Hoop stress and strain: } \quad \sigma_{\theta}=\varepsilon_{\theta} E, \quad \varepsilon_{\theta}=F_{r, \max } /\left\{2 \pi\left(S_{c u} E_{c u} \mu_{c u}+S_{\text {sus }} E_{\text {sus }}\right)\right\} \\
& \text { Bending stress and strain: } \sigma_{z}=E_{z} E \\
& E_{z}^{z}=F_{z, \max } L^{2} H_{s} /\left\{48 \pi\left(E_{c u} I_{c u} \mu_{c u}^{\prime}+E_{\text {sus }} I_{\text {sus }}\right)\right\}
\end{aligned}
$$

Allowable buckling load:

$$
P_{a}=0.807 \frac{2 \pi a E t^{2}}{r}\left\{\left(\frac{1}{1-v^{2}}\right)^{3} t^{2} / r^{2}\right\}^{1 / 4}
$$

where $\mathrm{E}$ is Young's modulus, $S$ the sectional area, $r$ the mean radius of coil, $I$ the moment of inertia of cross section, L the span between the supporting points, a the inner radius of coil, t the radial thickness of coil, $\mathrm{F}_{\mathrm{r}}$ and $\mathrm{F}_{\mathrm{z}}$ the radial and vertical electromagnetic force, $\mu$ and $\mu^{\prime}$ the effectiveness factors, $\nu$ Poisson's ratio.

\subsection{Heat Loss}

This subroutine calculates the heat loss caused by alternating magnetic field.

The following equations are utilized for the heat loss calculation [4], 1.e.

$$
\begin{array}{ll}
\text { Hysteresis loss: } & \mathrm{P}_{\mathrm{h}}=\frac{20}{3 \pi} \mathrm{J}_{\mathrm{C}} \mathrm{d} \quad \mathrm{B} \quad\left[1+\left(\mathrm{I}_{\mathrm{oP}} / \mathrm{I}_{\mathrm{C}}\right)^{2}\right\} \mathrm{A}_{\mathrm{sc}} \\
\text { Coupling loss: } & \mathrm{P}_{\mathrm{c}}=\frac{10^{-4}}{\mathrm{P}_{-4}} \mathrm{~B}^{2}\left(\frac{1}{2 \pi}\right)^{2} \mathrm{~A} \\
\text { Eddy current loss: } \mathrm{P}_{\mathrm{e}}=\frac{10^{-4}}{12 \rho} \mathrm{B}^{2} \mathrm{a}^{2} \mathrm{~A}
\end{array}
$$

where $B$ is the change rate of magnetic field with time, $I_{c}$ the critical current of superconductor, $A_{s c}$ the total area of the cross section of the superconducting filaments in the coil cross section, A the sum of sectional area of corresponding component in the coil cross section, a the width of corresponding component in a direction perpendicular to each component of $B$. 
4. Example of Coil Design using the System

This program system was tested by using IBM-3081 computer. Figure 4 shows an example of graphic display and output list of the dimensions of a conductor output in test run.

By using this system, the design of superconducting poloidal coils can be accomplished within 2 to 3 man days. Previously this took about 30 man-days.
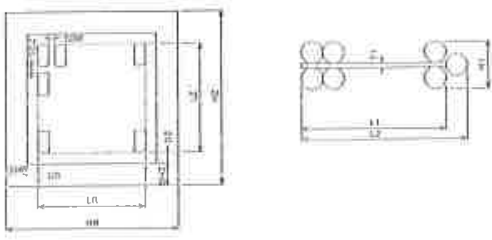

DIMENSION OF COIL SECTION (TYPE B)

PF COIL OF SC TOILAMAIC

\section{PF COIL OF SC TOKAMAK \\ DIMENSION OF COIL SECTION}

(TYPE B)

CASE
DIMENSION OF CASE
COIL
DIMENSION OF CONDUCTOR
CABLE
HEIGHT
LENGTH
HWIERIAL FOR REIMFORCEMENT
LWSULATOR
DIMEHSION TO EARTH

\begin{tabular}{|c|c|}
\hline $\begin{array}{l}\text { HR } \\
\text { SIIR }\end{array}$ & $\begin{array}{r}\quad 150.40(\mathrm{MH}) \\
=\quad 49.60(\mathrm{MM})\end{array}$ \\
\hline LR & $50.40(M M)$ \\
\hline HI & 5.50 (MM) \\
\hline LI & $28.00(114)$ \\
\hline TI & $1.50(\mathrm{MM})$ \\
\hline & $0.40(\mathrm{MM})$ \\
\hline SOR & $0.80(\mathrm{IM})$ \\
\hline
\end{tabular}

$\begin{aligned} H Z & =418.00(H H) \\ S H Z & =27.70(M M)\end{aligned}$

$L Z=358.00(\mathrm{WM})$

L2 - $30.00(\mathrm{Nit})$

$D Z=2.90(M+1)$

Fig. 4 Example of graphic display and output 1ist

5. Conclusion

A computer program system was developed for automatically designing the superconducting poloidal coils of tokamak. This system features:

(1) Labor and time for coil design is greatly reduced since the program system systematically deals with the design process such as the selection of required parameters for coil design, which up to now were manually derived.

(2) Quick response to any partial design modification since all final designs are stored in the data files.

(3) Very few engineers are able to accomplish a desirable design, which has required many engineers in various technical fields such as magnetic field analysis, superconductor design and structure analysis.

These features are very effective in case studies of poloidal coils relating the total design study of tokamak.

Reference

$/ 1 /$ SATOW, T., HATTORI, Y., IWAMOTO, M., "Optimum Current of Completely Stabilized Superconducting Coil", Proceedings National Meeting of IEEJ, (in Japanease), 632(1977)789.

/ $2 /$ MADDOCK, B. J., JAMES, G. B., NORRIS, W. T., "Superconductive Composites: Heat Transfer and Steady State Stabilization", Cryogenics 9, 261-273(1969).

/ / COLUMN RESEARCH COMMTTTEE OF JAPAN, "Summary of Elastic Stability", Tokyo: Corona, (in Japanease), 1960 .

/ 4 / JAPAN ATOMIC ENERGY RESEARCH INSTTTUTE, "Design Study of Superconducting Magnets For Tokamak Experimental Fusion Reactor (II)", JAERI-M8666, (in Japanease), 1979. 